\title{
Ventriculosinus shunt as a reliable option in the treatment of failed ventriculoperitoneal shunt: report of 19 cases and review of the literature
}

\author{
Xinxing $\mathrm{Li}^{1}$, Jihui Zheng ${ }^{2}$ \\ ${ }^{1}$ Department of Neurosurgery, Shengjing Hospital of China Medical University, Shenyang, China; ${ }^{2}$ Department of Radiology, The Fourth Affiliated \\ Hospital of China Medical University, Shenyang, China \\ Contributions: (I) Conception and design: X Li; (II) Administrative support: J Zheng; (III) Provision of study materials or patients: J Zheng; (IV) \\ Collection and assembly of data: X Li; (V) Data analysis and interpretation: X Li; (VI) Manuscript writing: Both authors; (VII) Final approval of \\ manuscript: Both authors. \\ Correspondence to: Jihui Zheng. Department of Radiology, The Fourth Affiliated Hospital of China Medical University, No. 4 Chongshan East Road, \\ Huanggu District, Shenyang 110032, China. Email: 53534529@qq.com.
}

\begin{abstract}
Background: The authors of this study aim to summarize their experience with lateral ventriculosinus shunt in the treatment of hydrocephalus patients and assess its clinical value.

Methods: Ventriculoperitoneal shunt was performed but failed due to obstruction and infection of the shunt. Lateral ventriculosinus shunt was inserted in 19 hydrocephalus patients by a Medtronic programmable shunt valve. All 19 patients suffered from either an obstruction or infection after ventriculoperitoneal shunting, while 11 cases had a peritoneal obstruction, 8 cases had an intracranial infection, and 1 case had an intra-abdominal infection. Among the 19 cases, there were 2 cases with obstructive hydrocephalus and 17 cases with communicating hydrocephalus.

Results: All clinical symptoms of 9 patients disappeared completely, while for 8 patients, noticeable improvement was accomplished, and only 2 patients showed no improvement. All 19 cases were followed up for 6 to 60 months. During the follow-up period, in 17 patients the shunt remained effective, while 2 cases underwent removal of the shunt due to low preoperative intracranial pressure and no significant changes in postoperative symptoms and ventricular size. During follow-up there was no thrombosis in the sagittal sinus, and no infection, over drainage, intracranial hemorrhage, or subdural effusion occurred.

Conclusions: We demonstrate that lateral ventriculosinus shunt is a reliable option for failed ventriculoperitoneal shunt hydrocephalus patients. Nevertheless, the effectiveness and possible complications of this method need further confirmation by observation in a study with a larger cohort.
\end{abstract}

Keywords: Hydrocephalus; Failed ventriculoperitoneal shunt; Ventriculosinus shunt

Submitted Apr 22, 2021. Accepted for publication Jun 08, 2021.

doi: 10.21037/apm-21-1202

View this article at: https://dx.doi.org/10.21037/apm-21-1202

\section{Introduction}

Hydrocephalus is a relatively common disease, which can be caused by numerous reasons. The main cause of hydrocephalus is the malabsorption of cerebrospinal fluid, which is mainly caused by intracranial infection; another is the blocked circulation of cerebrospinal fluid, which leads to the accumulation of local cerebrospinal fluid such as cerebral hemorrhage. Hydrocephalus may cause headaches, weakness of the lower limbs, unstable starting or standing gait, urinary incontinence and ataxia. Currently, while shunting is the main surgical therapy, there are more than 10 methods for the treatment of hydrocephalus. However, their therapeutic effects are unsatisfactory due to postoperative obstruction and infection of the 
shunt, along with further complications. Therefore, it is difficult for a clinician to choose the proper treatment. Ventriculosinus shunt is a feasible and reliable option for the treatment of hydrocephalus, especially for cases of failed ventriculoperitoneal shunt. Lateral ventriculosinus shunt path short, and lower incidence of drainage tube obstruction and infection. From January 2015 to January 2020, lateral ventriculosinus shunt was inserted in 19 failed ventriculoperitoneal shunt patients in our hospital, and we achieved excellent results which we report in this study.

We present the study in accordance with the STROBE reporting checklist (available at https://dx.doi.org/10.21037/ apm-21-1202).

\section{Methods}

The case inclusion criteria and general data were as follows: ventriculoperitoneal shunt was performed but failed due to obstruction and infection of the shunt. In the preoperative cerebrospinal fluid (CSF) test, the leucocyte count was normal, and the protein level was above normal, which was higher than $0.6 \mathrm{~g} / \mathrm{L}$ but lower than $1.0 \mathrm{~g} / \mathrm{L}$. By preoperative lumbar puncture, the intracranial pressure was measured higher than $15 \mathrm{cmH}_{2} \mathrm{O}$. Among the 19 patients, there were 8 males and 11 females. They were in the 15-56 age range and their average age was $37.41 \pm 6.72$. In all the 19 cases, the leucocyte count was normal, but the protein level was above normal in the preoperative CSF test. All 19 patients suffered from either an obstruction or infection after ventriculoperitoneal shunting, while 11 cases had a peritoneal obstruction, 8 cases had an intracranial infection, and 1 case had an intra-abdominal infection. All 11 patients with peritoneal obstruction underwent operation to adjust the position of the shunt several times. If the shunt was later considered ineffective, it was surgically removed. Patients with a confirmed diagnosis of an infection of their shunt device also got it surgically removed. Continuous lumbar cistern drainage was conducted until the leucocyte count was normal and the protein level was above normal but lower than $1.0 \mathrm{~g} / \mathrm{L}$ in a preoperative CSF test. Among the 19 cases, there were 2 cases with obstructive hydrocephalus and 17 cases with communicating hydrocephalus.

This research was approved by the Ethics Committee of the Shengjing Hospital of China Medical University. All family members of the patients had signed the informed consent for publication of this study and any accompanying images. All procedures performed in studies involving human participants were in accordance with the Helsinki
Declaration (as revised in 2013).

\section{Operation method}

A Medtronic programmable shunt valve was adopted in this group. The patients were in the supine position under local or general anesthesia. Centered on the routine frontal puncture point $(2.5 \mathrm{~cm}$ within the hairline and $2.5 \mathrm{~cm}$ by the midline) in the lateral ventricle, a straight scalp incision was made in the frontal hairline. The skull external lamina was exposed, and drill a bone hole. The ventricular end of the shunt was then placed into the lateral ventricle, and the depth was between 4 and $6 \mathrm{~cm}$. The outflow of CSF in the shunt was directly verified by visual confirmation. Subsequent to the successful puncture, the ventricular end of the shunt was immediately connected to a pressure measuring tube. Using the cerebral ventricle plane as the baseline, the intraventricular pressure was measured. The initial pressure value of the shunt valve was set at $5 \mathrm{cmHg}$ lower than the measured pressure. Another incision was made at the top of the sagittal sinus, in the forward and backward direction, about $3 \mathrm{~cm}$ in length. Enough space was left between the 2 incisions to place the shunt. First, the skull external lamina was exposed, and a second bone hole was drilled at the sagittal suture to expose the sagittal sinus. A $1 \mathrm{~mL}$ syringe was carefully penetrated into the superior sagittal sinus. In order to confirm the correct location of the needle, the syringe's plunger was drawn backwards, until venous blood was visible. Only then the needle was carefully inserted a further $0.5 \mathrm{~cm}$. Afterwards, as a final verification of the correct location of the needle at the superior sagittal sinus, the syringe's plunger was drawn backwards one more time, until venous blood was visible. Subsequently, a 3-mm long arc incision was made along the puncture points at the superior sagittal sinus wall. The sagittal sinus end of the shunt tube was inserted into the incision and retained about 6-8 $\mathrm{cm}$ in the sagittal sinus. The shunt pump was placed in the substratum of galea aponeurotic between the two incisions and connected to the ventricular end of the shunt. A 20-mL syringe was used to inject a physiological salt solution into the sagittal sinus shunt to remove the blood remaining from the puncture procedure. After confirming that there was no contraflow of intrasinus blood, the shunt pump was linked to the sagittal sinus shunt. Generally, the point of insertion of the shunt tube into the sagittal sinus showed no obvious bleeding, as the shunt tube is closely bound to the puncture hole. A small gelatin sponge was inserted for compressing hemostasis. Ultimately, we checked the shunt 

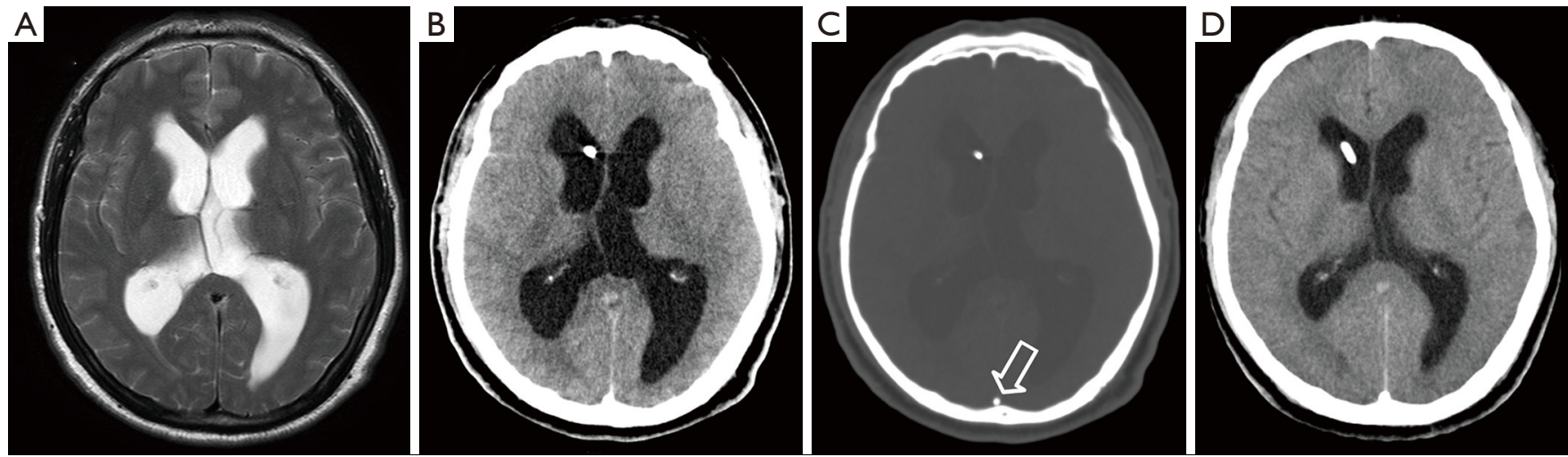

Figure 1 Comparison of ventricular size before and after operation. (A) Preoperative MR T2WI showed bilateral ventricular dilatation; (B) CT scan on the second day after operation showed that the shunt was located in the frontal angle of the lateral ventricle; (C) the shunt in the sagittal sinus was seen through CT bone window examination on the second day after the operation (as indicated by the arrow); (D) CT scan 6 months after the operation showed that bilateral ventricles were significantly smaller than before the operation.
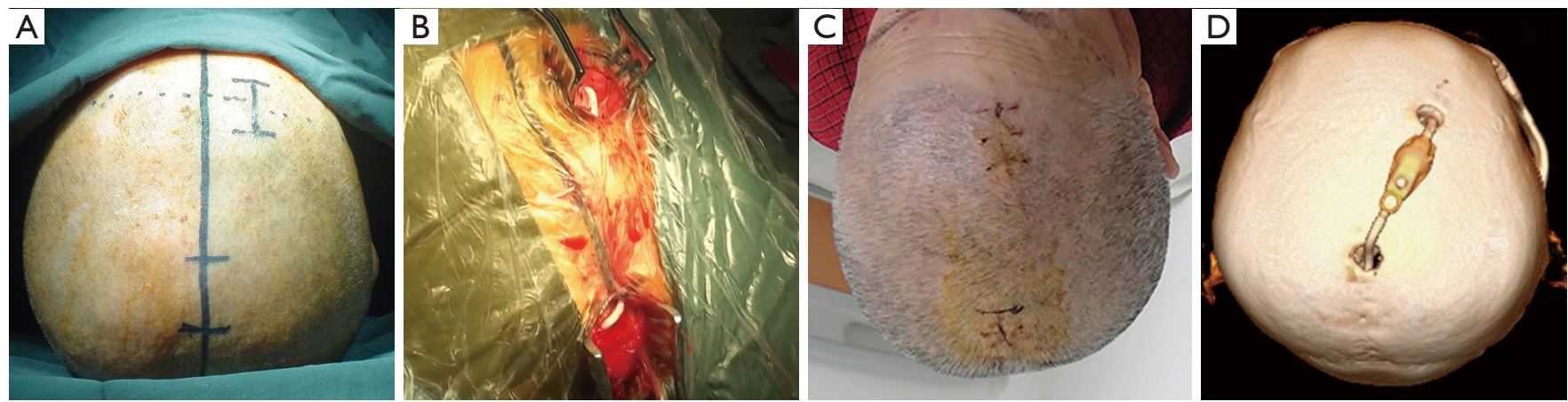

Figure 2 Straight scalp incision and postoperative three-dimensional CT reconstruction of the shunt tube. (A) Straight scalp incision of the routine frontal puncture point and the top of the sagittal sinus; (B) the ventricular end and the sagittal sinus end of the shunt were placed into the lateral ventricle and the sagittal sinus respectively; (C) scalp incision has a good prognosis; (D) postoperative three-dimensional CT reconstruction of the shunt tube.

pump and pressed the fluid storage capsule to confirm the normal function of the shunt device, then finally sutured and fixed the scalp, and finished the operation.

\section{Statistical analysis}

The SPSS19.0 statistical analysis software was used for analysis and processing. Normally distributed measurement data were expressed as mean \pm standard deviation, and count data were expressed as percentages.

\section{Results}

Brain CT re-examination of all patients 48 hours after surgery showed that the ventricles were smaller than before surgery (Figure 1). The ventricle ends of the shunts were located in the frontal lobes of the lateral ventricles, and the ends were located in the superior sagittal sinus (Figure 2). When the 19 patients were discharged from our hospital, the clinical symptoms were significantly relieved or even disappeared completely in 9 cases. For 8 cases, improvement was achieved, while the remaining 2 cases showed no significant improvement. The patients in our group were followed up for 6-60 months $(39.11 \pm 6.28)$. During the follow-up period, the clinical manifestations and imaging results were used as the basis for regulating the pressure of the shunt valve. If there was no change, the pressure of the shunt valve was reduced. After the pressure adjustment, 
the patients were revisited once a month until the clinical symptoms were significantly relieved or completely disappeared. During the follow-up period, in 17 patients the shunt remained effective, while 2 cases underwent removal of the shunt due to low preoperative intracranial pressure and no significant changes in postoperative symptoms and ventricular size. Therefore, a ventriculo-bladder shunt was placed instead at an optional date. No venous sinus thrombosis, shunt infection, excessive shunt, intracranial hemorrhage, or subdural effusion occurred during the follow-up period.

\section{Discussion}

Hydrocephalus is a common disease in neurosurgery with complex etiology and pathogenesis. With the progress in medicine, the diagnosis and treatment of hydrocephalus have been enriched, and the treatment level of hydrocephalus has been constantly improved. At present, operative treatment is the most commonly used method to treat hydrocephalus. According to different causes, the most commonly used operative treatment methods are CSF shunt and endoscopic third ventriculostomy (ETV) (1-3). Currently, the most commonly used shunting is the ventriculoperitoneal shunt. Compared with the ventriculosinus shunt and ventriculoatrial shunt, the ventriculoperitoneal shunt has certain advantages. The drainage of CSF to the abdominal cavity with a large space has strong absorption capacity for CSF and a simple operation. In recent years, the invention of various new drainage devices and the improvement of surgical techniques have made the ventriculoperitoneal shunt widely used, but due to the limitation of catheter materials and techniques, the ventriculoperitoneal shunt will inevitably produce some complications, which will affect the surgical results.

Shunt obstruction is a common cause of shunt failure. The incidence of shunt obstruction was previously $28 \%$. Currently, despite all improvements, the lowest reported obstruction rate is $15 \%(4,5)$. In addition to the obstruction of brain tissue debris and blood clots, the location of the end of the ventricle may be another major cause of proximal tube obstruction. For example, the length of the ventricle end in the ventricle was not appropriate, or the end of the ventricle was relatively too short, and the lateral orifice of the shunt tube was not completely inserted into the ventricle. Also, there is a possibility that the end of the ventricle was relatively long and entered the third ventricle with the interventricular foramen, or it was wrapped by the ventricular choroid plexus and thus obstructed drainage. Distal obstruction was usually caused by the placement of too long or too short intraperitoneal shunts, resulting in its folding or angulation. Another complication was peripheral inflammation and foreign body reactions surrounding the greater omentum, resulting in the obstruction of the intraperitoneal shunt. Postoperative infection is a more serious complication. Previously, the first infection rate of the drainage tube was $15 \%$, and the second infection rate was about $26 \%(6,7)$. Currently, despite improvements in all aspects, the lowest infection rate is still $5 \%(5,6)$. The manifestations of infection after shunting vary. The gold standard for diagnosis is to extract CSF for culture, but the results of culture are mostly negative. Indirect manifestations of intracranial infection include persistent fever, headache, vomiting, convulsions, and a stiff neck. Laboratory values include an increased white blood cell count and C-reactive protein content in the blood. When performing a CSF assay, white blood cell count and protein levels are increased, while glucose levels are decreased (6). Abdominal end infection manifests as continuous fever, abdominal pain, positive peritoneal stimulation, and increased white blood cell count. Once the infection after shunting is diagnosed, antibiotics should be used according to the common bacterial spectrum, and then adjusted according to the results of CSF culture. The shunt device should be surgically removed as soon as possible, along with draining any remaining pus. Studies have shown that $26 \%$ of patients with an infection after ventriculoperitoneal shunt who underwent ventriculoperitoneal shunt again had a recurrent infection (6). In our group, there were 9 patients with infections after peritoneal shunt, and no recurrent infection occurred after repeat ventriculosinus shunt. In recent years, it has been reported abroad that lateral ventriculosinus shunt was used to treat hydrocephalus with satisfactory postoperative results, and no venous sinus thrombosis occurred during the observation period. Autopsy was performed on some deceased patients, and no thrombosis was found in the venous sinus $(8,9)$. In this study, 19 cases of hydrocephalus were treated by lateral ventriculosinus shunt with a satisfactory curative effect, and no venous sinus thrombosis and other complications occurred during the follow-up.

The lateral ventriculosinus shunt is consistent with the physiological process of introducing CSF directly from the ventricle into the blood circulation. Under physiological conditions, CSF circulation enters the blood circulation from the ventricle through the subarachnoid space to the 
venous sinus or is absorbed from the subarachnoid space into the blood circulation through the perivascular space and extracellular space of the central nervous tissue. According to the therapeutic results of patients in this group, lateral ventriculosinus shunt has a shorter duration, which reduces the chance of shunt obstruction and infection, and especially avoids the greater omentum's encirclement and blockage of the intraperitoneal shunt and avoids the need to adjust the length of the shunt due to the increasing duration. The surgical area, and therefore, the injury, is small, and also the incision is completely concealed in the hairline, which is not only cosmetically pleasing, but also avoids subcutaneous and open wounds caused by the shunt tube.

As the lateral ventriculosinus shunt directly diverts the CSF into the blood system, more attention than normal should be paid to strict aseptic operation conditions, and effective antibiotics should be used during and after the operation as a standard procedure of care. The shunt device is firmly fixed to prevent falling off. Sagittal sinus incision should not be too large to prevent tearing and bleeding afterwards. The shunt tube should be smooth through the bone pore margin, without compression, so as to avoid the influence of the shunt tube being folded on an obstruction. If the preoperative CSF test indicates an infection or increased protein levels, lumbar cistern drainage should be performed first until the CSF cell count is normal. Due to the absence of greater omentum encirclement and blockage of the shunt tube, the CSF protein level was slightly higher than normal. In this group of patients, the preoperative CSF protein level was greater than $0.6 \mathrm{~g} / \mathrm{L}$, but less than $1.0 \mathrm{~g} / \mathrm{L}$. No cases of tube blockage occurred during the follow-up period. Additionally, preoperative intracranial pressure should be measured. Since the venous pressure in the superior sagittal sinus was $5-10 \mathrm{cmH}_{2} \mathrm{O}$, and the lowest pressure in the Medtronic adjustable shunt tube was $5 \mathrm{cmH}_{2} \mathrm{O}$, the high cranial pressure hydrocephalus shunt had a better effect. It has been reported that low cranial pressure or normal pressure ventriculosinus shunt has a poor prognosis $(10,11)$. In this group, 2 cases were cured after ventriculo-bladder shunt due to low preoperative intracranial pressure and no significant changes in postoperative symptoms and ventricular size. When the sagittal sinus was punctured, the front crack of the shunt tube should not be opened as far as possible, so as to avoid blood flowing back into the tube and forming a thrombus to block the tube. Once the blood in the sinus flows back into the tube, the tube is flushed repeatedly with a sufficient amount of physiological salt solution until no blood in the sinus flows out.

ETV is suitable for obstructive hydrocephalus above the 4th ventricle outlet, especially for aqueduct stenosis or other obstructive hydrocephalus below the other 3 ventricles $(12,13)$. Its trauma is small, the infection rate is low, and the operation result is more consistent with normal CSF physiological circulation. Compared with the shunt, ETV has the following advantages: (I) there are no complications due to shunt device placement; (II) it is basically approximate to the physiological status of CSF circulation; (III) lower surgical trauma and shorter duration; (IV) a variety of methods can be used at the same time to improve the curative effect, such as choroid electrocoagulation and aqueductal stenosis balloon angioplasty, among others. However, this operation also has some limitations, and there are inevitable postoperative complications. General complications of ETV include postoperative intracranial hemorrhage, fever, meningitis, CSF leakage, transient diabetes insipidus, memory impairment, oculomotor nerve palsy, postoperative secondary infection, and epilepsy, among others. Severe complications include fatal subarachnoid hemorrhage, traumatic aneurysms in the basilar artery, and postoperative fistula closure (14). It has been ascertained that ETV treatment for obstructive hydrocephalus is the most effective, followed by aqueduct stenosis, but the success rate for hydrocephalus was very low due to bleeding and inflammation (15). It is believed that with the continuous development of endoscopic technology, postoperative complications will be gradually reduced, and its application prospects will be broader. Any kind of operation method is not perfect and has its own limitations. The choice of operation method should be made according to the patient's age, etiology, classification, and other specific circumstances. ETV treatment for obstructive hydrocephalus is obviously superior to shunt surgery in terms of surgical effect, postoperative complications, and the postoperative rehabilitation of patients, but cannot completely replace shunt surgery. Ventriculoperitoneal shunt is still the first choice for CSF shunt. For patients with high cranial pressure hydrocephalus, or patients not suitable for peritoneal shunt, lateral ventriculosinus shunt should be their reliable option.

In conclusion, lateral ventriculosinus shunt can be used as a surgical method for the treatment of refractory hydrocephalus due to a short shunt path and a low incidence of drainage tube obstruction and infection. Because of the small sample size and relatively short followup time, the long-term efficacy and complication rate of 
lateral ventriculosinus shunt still remains to be further demonstrated by a large-scale case study.

\section{Acknowledgments}

Funding: This work is supported by grants from the Liaoning Science and Technology Plan Project (No. 2018225094), and Natural Science Foundation of Liaoning Province (No. 2020-MS-165).

\section{Footnote}

Reporting Checklist: The authors have completed the STROBE reporting checklist. Available at https://dx.doi. org/10.21037/apm-21-1202

Data Sharing Statement: Available at https://dx.doi. org/10.21037/apm-21-1202

Conflicts of Interest: Both authors have completed the ICMJE uniform disclosure form (available at https://dx.doi. org/10.21037/apm-21-1202). The authors have no conflicts of interest to declare.

Ethical Statement: The authors are accountable for all aspects of the work in ensuring that questions related to the accuracy or integrity of any part of the work are appropriately investigated and resolved. This research was approved by the Ethics Committee of the Shengjing Hospital of China Medical University. All procedures performed in studies involving human participants were in accordance with the Helsinki Declaration (as revised in 2013). Written informed consent was obtained from the patient for publication of this study and any accompanying images.

Open Access Statement: This is an Open Access article distributed in accordance with the Creative Commons Attribution-NonCommercial-NoDerivs 4.0 International License (CC BY-NC-ND 4.0), which permits the noncommercial replication and distribution of the article with the strict proviso that no changes or edits are made and the original work is properly cited (including links to both the formal publication through the relevant DOI and the license). See: https://creativecommons.org/licenses/by-nc-nd/4.0/.

\section{References}

1. Jayanth A, Benabbas R, Chao J, et al. Diagnostic modalities to determine ventriculoperitoneal shunt malfunction: A systematic review and meta-analysis. Am J Emerg Med 2021;39:180-9.

2. Kontojannis V, Papadopoulos E, Ydreos J, et al. Endoscopic Third Ventriculostomy: A Single-Center Experience in Greece. Surg J (N Y) 2020;6:e71-e76.

3. Lu L, Chen H, Weng S, et al. Endoscopic Third Ventriculostomy versus Ventriculoperitoneal Shunt in Patients with Obstructive Hydrocephalus: Meta-Analysis of Randomized Controlled Trials. World Neurosurg 2019;129:334-40.

4. Docter TA, Patel BH, Brennan JJ, et al. Utility of Shunt Series in the Evaluation of Ventriculoperitoneal Shunt Dysfunction in Adults. J Emerg Med 2020;58:391-7.

5. Sweeney J, Zyck S, Tovar-Spinoza Z, et al. EvidenceBased Perioperative Protocol for Ventriculoperitoneal Shunt Infection Reduction at a Single Institution. World Neurosurg. 2019;128:e814-e822.

6. López-Sánchez C, Rozas-Muñoz E, Mir-Bonafé JF. Ventriculoperitoneal Shunt Infection. JAMA Dermatol 2020;156:1366.

7. Rocque BG, Waldrop RP, Shamblin I, et al. Shunt failure clusters: an analysis of multiple, frequent shunt failures.

J Neurosurg Pediatr 2020. [Epub ahead of print]. doi: 10.3171/2020.7.PEDS20199.

8. Baert EJ, Dewaele F, Vandersteene J, et al. Treating Hydrocephalus with Retrograde Ventriculosinus Shunt: Prospective Clinical Study. World Neurosurg. 2018;118:e34-e42.

9. Vandersteene J, Baert E, Schauvliege S, et al. A nonhydrocephalic goat experimental model to evaluate a ventriculosinus shunt. Lab Anim 2018;52:504-14.

10. Oliveira MF, Teixeira MJ, Reis RC, et al. Failed Ventriculoperitoneal Shunt: Is Retrograde Ventriculosinus Shunt a Reliable Option? World Neurosurg 2016;92:445-53.

11. Vandersteene J, Baert E, Planckaert GMJ, et al. The influence of cerebrospinal fluid on blood coagulation and the implications for ventriculovenous shunting. J Neurosurg 2018. [Epub ahead of print]. doi: 10.3171/2017.11.JNS171510.

12. Sarmast A, Khursheed N, Ramzan A, et al. Endoscopic Third Ventriculostomy in Noncommunicating Hydrocephalus: Report on a Short Series of 53 Children. Asian J Neurosurg 2019;14:35-40.

13. Tiefenthaler W, Burtscher J, Moser PL, et al. Intraventricular Pressure in Non-communicating 
Hydrocephalus Patients Before Endoscopic Third Ventriculostomy. Open Med (Wars) 2019;14:909-12.

14. Luther E, McCarthy D, Sedighim S, et al. Endoscopic third ventriculostomy inpatient failure rates compared with shunting in post-hemorrhagic hydrocephalus of prematurity. Childs Nerv Syst 2020;36:559-68.

Cite this article as: $\mathrm{Li} \mathrm{X}$, Zheng J. Ventriculosinus shunt as a reliable option in the treatment of failed ventriculoperitoneal shunt: report of 19 cases and review of the literature. Ann Palliat Med 2021;10(7):7627-7633. doi: 10.21037/apm-21-1202
15. Wang Q, Cheng J, Zhang S, et al. Prediction of endoscopic third ventriculostomy (ETV) success with preoperative third ventricle floor bowing (TVFB): a supplement to ETV success score. Neurosurg Rev 2020;43:1575-81.

(English Language Editor: C. Betlazar-Maseh) 